\title{
DERIVATIVES OF HIGHER ORDER AS SINGLE LIMITS*
}

BY PHILIP FRANKLIN

1. Introduction. If a function has an $n$th derivative at a point, the value of this derivative may be calculated by taking the limit of a suitable quotient involving the $n$th difference, as the fundamental difference for the independent variable approaches zero. However, the limit of this quotient may exist without the function having the corresponding derivative. The principal result of this paper deals with the expression for unevenly spaced points, which reduces to the quotient mentioned above when the points are equidistant. It is shown that the existence of the limit of this expression, when the points close down in any way whatever to a fixed point, is a necessary and sufficient condition for the function to have an $n$th derivative throughout some neighborhood of the fixed point, continuous at the point. Some applications to finite Taylor developments are considered. A condition for the mere existence of the derivative is also given.

2. Earlier Results. A precise statement of the theorem on $n$th differences, $\dagger$ as proved by de la Vallee-Poussin, is that

$$
\lim _{h \rightarrow 0} \frac{\Delta^{n} y}{h^{n}}=f^{(n)}\left(x_{0}\right),
$$

if $y=f(x)$ has an $n$th derivative at $x_{0}$, where $\Delta^{n} y$ is the $n$th difference defined by the formulas

$$
\begin{aligned}
\Delta^{n} f(x) & =\Delta^{n-1} f(x+h)-\Delta^{n-1} f(x), \\
\Delta f(x) & =f(x+h)-f(x) .
\end{aligned}
$$

The corresponding expression for unevenly spaced points is, except for a numerical factor, $n !$,

(2) $A_{n, k}=\sum_{j=1}^{n} \frac{f\left(x_{j}\right)}{\left(x_{j}-x_{1}\right) \cdots\left(x_{j}-x_{j-1}\right)\left(x_{j}-x_{j+1}\right) \cdots\left(x_{j}-x_{n+1}\right)}$,

* Presented to the Society, December 27, 1933.

$\dagger$ de la Vallée-Poussin, Cours d'Analyse Infinitésimale, vol. 1, 1921, p. 73. 
as is seen by comparing Newton's and Lagrange's interpolation formulas. It is sometimes convenient to write this in terms of determinants:

$$
A_{n, k}=\frac{\left|f\left(x_{j}\right), x_{j}^{n-1}, \cdots, x_{j}, 1\right|}{\left|x_{j}^{n}, x_{j}^{n-1}, \cdots, x_{j}, 1\right|} .
$$

We have written only the $j$ th row, $(j=1,2, \cdots, n+1)$, for the rest.

It has been shown by the writer* that if $y=f(x)$ has an $n$th derivative at $a$, continuous in some neighborhood of $x_{0}$, then as the points close down to $a$, that is,

$$
\lim _{k \rightarrow \infty} \epsilon_{k}=0,\left|x_{j}-a\right|<\epsilon_{k},
$$

we shall have

$$
f^{(n)}(a)=\lim _{k \rightarrow \infty} n ! A_{n, k} .
$$

A similar result was proved $\dagger$ for the case of a complex independent variable, with the points closing down in any way, and $f(x)$ an analytic function.

The limit using the $n$th difference is too broad to be used as a definition of a derivative. For this limit may exist without there being a derivative. An example is the function $y=b x$, when $x$ is rational, $y=c x$, when $x$ is irrational. If $a=0$, the points 0 , $h, 2 h, \cdots, n h$, are either all rational or all irrational, so that at $0, \Delta^{2} y=0$, and $\lim \Delta^{2} y / h^{2}=0$. However, the function fails to have a first derivative at the origin, and hence has no second derivative there.

Again, as a definition of mere existence of a derivative, the use of $A_{n, k}$ with points closing down arbitrarily is unsatisfactory since it fails to give correct results unless the derivative is continuous at the point $a$ considered. For, if the $n$ points are allowed to come together at a point near $a$, in the proper way, for example with equal spacing, they will give the derivative at this point. If the derivative is not continuous at $a$, this derivative will not approach the value at $a$, when the nearby point approaches $a$.

* Transactions of this Society, vol. 28 (1926), p. 403.

$\dagger$ This Bulletin, vol. 33 (1927), p. 465. 
3. Condition for a Continuous nth Derivative. Consider a sequence of sets of $n+1$ points. Call the $k$ th set $S_{k}$, and let its elements satisfy (4). Then we shall say that the set $S_{k}$ closes down on $a$. For each set we may form $A_{n, k}$ by (2), or (3). We may now formulate* the following theorem.

THEOREM 1. The existence of a unique limit for $A_{n, k}$ as the points of $S_{k}$ close down on a in an arbitrary manner is a necessary and sufficient condition for $f(x)$ to have an nth derivative $f^{n}(a)$, continuous at a. The value of the derivative is given by (5).

'That the condition is necessary $\dagger$ follows from Rolle's theorem and Lagrange's interpolation formula. For

(6) $F(x)$

$$
=\sum_{j=1}^{n} \frac{\left(x-x_{1}\right) \cdots\left(x-x_{j-1}\right)\left(x-x_{j+1}\right) \cdots\left(x-x_{n+1}\right)}{\left(x_{j}-x_{1}\right) \cdots\left(x_{j}-x_{j-1}\right)\left(x_{j}-x_{j+1}\right) \cdots\left(x_{j}-x_{n+1}\right)} f\left(x_{j}\right)
$$

is a polynomial of the $n$th degree agreeing with $f(x)$ at the points of $S_{k}$. Hence, $f(x)-F(x)$ vanishes at $n+1$ points, and by successive application of Rolle's theorem its $n$th derivative vanishes at some point $X$, interior to the interval whose end points are the extreme points of $S_{k}$. That is,

$$
F^{(n)}(X)=f^{(n)}(X)
$$

But from (2) and (6),

$$
F(x)=A_{n, k} x^{n}+P_{n-1}(x),
$$

where $P_{n-1}(x)$ is a polynomial of the $(n-1)$ th degree, so that

$$
F^{(n)}(X)=n ! A_{n, k} .
$$

Since the points of $S_{k}$ are closing down on $a, X$ approaches $a$ as a limit, so that from the continuity of $f^{(n)}(x)$,

$$
\lim _{k \rightarrow \infty} n ! A_{n, k}=\lim _{X \rightarrow a} f^{(n)}(X)=f^{(n)}(a) .
$$

This proves the necessity of the condition.

* Hassler Whitney, Transactions of this Society, vol. 36 (1934), p. 369. This paper contains certain similar results of this type, involving uniformity conditions.

$\dagger$ Transactions of this Society, vol. 28 (1926), p. 403. 
We shall prove the sufficiency of the condition by mathematical induction. For $n=1$, we have

$$
A_{1, k}=\frac{f\left(x_{2}\right)-f\left(x_{1}\right)}{x_{2}-x_{1}} .
$$

By taking $x_{1}=a$, and letting $x_{2}$ approach $a$, we see that $f^{\prime}(a)$ exists. Again, since the limit must exist when $x_{2}$ approaches $x_{1}$, and then if we let $x_{2}$ remain coincident with $x_{1}$ and approach $a$, we have

$$
f(a)=\lim _{x_{1} \rightarrow a} \lim _{x_{2} \rightarrow x_{1}} A_{1, k}=\lim _{x_{1} \rightarrow a} f^{\prime}\left(x_{1}\right),
$$

so that $f^{\prime}\left(x_{1}\right)$ exists for $x_{1}$ near $a$, and is continuous at $a$. We accordingly assume as the basis of the induction that the condition is sufficient for $n=1,2, \cdots, N$. Now consider $A_{n, k}$, and to simplify the notation change the origin so that $a=0$. Put $x_{1}=x$, $x_{2}=0$, and let $x$ approach zero. Then all the terms in the sum (2) for $A_{n, k}$ except the first two approach limits, and the first two may be written:

$$
\begin{aligned}
& \frac{f(x)-f(0)}{x} \frac{1}{\left(x-x_{3}\right) \cdots\left(x-x_{n+1}\right)} \\
& \quad+\frac{f(0)}{x}\left[\frac{1}{\left(x-x_{3}\right) \cdots\left(x-x_{n+1}\right)}-\frac{1}{\left(-x_{3}\right) \cdots\left(-x_{n+1}\right)}\right] .
\end{aligned}
$$

As the second of these approaches a limit for $x$ approaching zero, and the product does, we have shown the existence of

$$
f^{\prime}(0)=\lim \frac{f(x)-f(0)}{x} .
$$

We now define a continuous function $v(x)$ by the equations

$$
\begin{aligned}
& f(x)=f(0)+x v(x), \quad(x \neq 0), \\
& v(0)=f^{\prime}(0) .
\end{aligned}
$$

If we calculate $A_{n, k}$ by (3), for $x_{n+1}=0$, and make use of (14), we find, after reduction,

$$
A_{n, k}=\frac{\left|v\left(x_{j}\right), x_{j}{ }^{n-2}, \cdots, x_{j}, 1\right|}{\left|x_{j}^{n-1}, x_{j}{ }^{n-2}, \cdots, x_{j}, 1\right|}, \quad(j=1,2, \cdots, n)
$$


As this has the same form as (3), with $f(x)$ replaced by $v(x)$, and $n$ by $n-1$, by the basis of our induction, it follows that

$$
\lim N ! A_{N+1, k}=v^{(N)}(0),
$$

and that $v^{(N)}(x)$ exists in some neighborhood of $a=0$, and is continuous at this point. The derivatives of $v(x)$ of lower order have this same property.

By differentiating (14), we find

$$
f^{(n)}(x)=x v^{(n)}(x)+n v^{(n-1)}(x) .
$$

This is in the first instance only established for $x \neq 0$, but follows for $x=0$ from the continuity* of the derivatives of $v(x)$, which shows that for $n \leqq N, f^{(n)}(0)$ exists and is given by

$$
f^{(n)}(0)=n v^{(n-1)}(0) \text {. }
$$

Thus

$$
\frac{f^{(N)}(x)-f^{(N)}(0)}{x}=v^{(N)}(x)+N \frac{v^{(N-1)}(x)-v^{(N-1)}(0)}{x},
$$

and on letting $x$ approach zero, we show the existence of

$$
f^{(N+1)}(0)=(N+1) v^{N}(0) .
$$

Comparison with (17) shows that

$$
\lim (N+1) ! A_{N+1, k}=f^{(N+1)}(0)
$$

for the special approach used, and hence for any approach since the limit is assumed to be unique. In particular, if we first let the $(N+1)$ points of $S_{k}$ coalesce at $x_{1}$ near $a=0$, and then remaining coincident with $x_{1}$ approach $a=0$, we shall have

$$
f^{(N+1)}(0)=\lim _{x_{1} \rightarrow 0} f^{(N+1)}\left(x_{1}\right),
$$

since the point $x_{1}$ may be taken as the 0 in (22), when sufficiently near $a$ for the limit to exist. This establishes the continuity of $f^{(N+1)}(x)$ at 0 , and so proves the sufficiency of the condition.

4. Taylor Developments. If a function has an $n$th derivative at a point, $f^{(n)}(a)$, and we form its Taylor development

* de la Vallée-Poussin, loc. cit., p. 127, number 5. 


$$
\begin{aligned}
f(a+h)=f(a) & +\frac{h}{1} f^{\prime}(a)+\cdots \\
& +\frac{h^{n-1}}{(n-1) !} f^{(n-1)}(a)+\frac{h^{n}}{n !} M(h) ;
\end{aligned}
$$

then, as shown by W. H. Young, ${ }^{*}$

$$
\lim _{h \rightarrow 0} M(h)=f^{(n)}(a) \text {. }
$$

We may have a development of this form

$$
f(a+h)=A_{0}+\frac{h}{1} A_{1}+\cdots+\frac{h^{n-1}}{(n-1) !} A_{n-1}+\frac{h^{n}}{n !} M(h)
$$

with

$$
\lim _{h \rightarrow 0} M(h)=M(0),
$$

without $f(x)$ having an $n$th derivative at $x=a$.

In fact, such a development implies nothing as to the derivatives of $f(x)$ beyond the fact that $f^{\prime}(a)=A_{1}$. This is shown by the following example:

$$
f(x)=x^{n+1} \sin x^{-n-1}, \quad x \neq 0, \quad f(0)=0 .
$$

There is a development about the origin, of the form (25), with

$$
A_{1}=A_{2}=\cdots=M(0)=0, \quad M(h)=n ! h \sin h^{-n-1} .
$$

However $f^{\prime}(x)$ is discontinuous at 0 , so that there is no second derivative.

We give a condition for a development of type (25) in the following theorem.

THEOREM 2. The existence of the limit $A_{n, k}$ for all sets of points closing down to $a$ in such a way that a bound $K$ exists such that

$$
\left|x_{j}-a\right| \leqq K\left|x_{i}-x_{i}\right|, \quad(i \neq j),
$$

is a necessary and sufficient condition for $f(x)$ to have a Taylor development of the nth order about the point a.

*W. H. Young, The Fundamental Theorems of the Differential Calculus, Cambridge Tract, No. 10 (1910), p. 16; see de la Vallée Poussin, loc. cit., p. 77. 
It may be noted that the condition (27) prevents the points from having mutual distances small compared with their distances from $a$. In particular, two points can not coalesce unless they both coincide with $a$.

To prove the necessity, we simplify the notation by taking $a=0$, so that our assumed development is

$$
f(x)=A_{0}+\frac{x}{1} A_{1}+\cdots+\frac{x^{n-1}}{(n-1) !} A_{n-1}+\frac{x^{n}}{n !} M(x) .
$$

Now calculate $A_{n, k}$ by using (3) and (28) for the functions $f\left(x_{j}\right)$ in the numerator. The result is

$$
\begin{aligned}
& n ! A_{n, k}=\frac{\left|x_{j}^{n} M\left(x_{j}\right), x_{j}^{n-1}, \cdots, x_{j}, 1\right|}{\left|x_{j}^{n}, x_{j}^{n-1}, \cdots, x_{j}, 1\right|}=M(0) \\
& \quad+\sum_{j=1}^{n+1} \frac{x_{j}^{n}\left[M\left(x_{j}\right)-M(0)\right]}{\left(x_{j}-x_{1}\right) \cdots\left(x_{j}-x_{j-1}\right)\left(x_{j}-x_{j+1}\right) \cdots\left(x_{j}-x_{n+1}\right)} .
\end{aligned}
$$

From (27) we have $\left|x_{j}\right| \leqq K\left|x_{j}-x_{i}\right|,(i \neq j)$, so that each term in the summation of (29) is dominated by $K^{n}\left|M\left(x_{j}\right)-M(0)\right|$ and approaches zero when $M\left(x_{j}\right) \rightarrow M(0)$. If any point $x_{j}$ reaches $a=0$, the corresponding term does not appear in the summation. Thus, if there is a development (28), when (27) holds,

$$
\lim n ! A_{n, k}=M(0) \text {. }
$$

For the sufficiency, we use the reasoning previously given to establish (13), which is valid since the conditions there used are compatible with (27). We define $v(x)$ by (14) and (15), and consider (16). Now use mathematical induction. For $n=1$, the existence of a derivative implies the existence of a development (28). Assume the theorem holds for $n=1,2, \cdots, N-1$. Then from this, applied to $v(x)$, we have

$$
\frac{f(x)-f(0)}{x}=v(x)=B_{0}+B_{1} x+\cdots+\frac{x^{N-1}}{(N-1) !} B(x)
$$

and from this

$$
f(x)=f(0)+B_{0} x+B_{1} x^{2}+\cdots+\frac{x^{N}}{N !} N B(x),
$$

which proves the result for $n=N$, completing the induction. 
COROLLARY. If the function $f(x)$ has an nth derivative at $a$, then as $S_{k}$ closes down to a, subject to (27),

$$
\lim n ! A_{n, k}=f^{n}(a) \text {. }
$$

For, under the hypothesis, there is a development given by (23) and (24), and the result follows from (30).

5. Contact. The order of contact of two curves is usually stated in terms of derivatives. However, as the derivatives are merely used to get the Taylor development, if we have a Taylor development at hand it may be used to define order of contact. Thus, the example previously mentioned,

$$
y=x^{n+1} \sin x^{-n-1}, \quad x \neq 0 ; \quad y=0, \quad x=0,
$$

is a curve having contact of the $n$th order with the $x$ axis at the origin.

Similarly, certain other geometric concepts may be reduced to that of contact. Thus, in this sense, the curve

$$
y=2 k x^{2}+x^{3} \sin \frac{1}{x}
$$

has curvature $k$ at the origin. That is, it has second order contact with a circle of radius $1 / k$, the circle of curvature. There is no second derivative at the origin. However, by Theorem 2, if a circle is passed through three points on the curve, which close down to the origin subject to the condition (27), it will approach the circle of curvature.

In a space where distances, rather than coordinates, are given, it would be natural to replace $(27)$ by a similar condition on the distances. This would lead to an intrinsic definition of curvature more general than that of Menger, ${ }^{*}$ based on the expression for the radius of the circle through the three points, allowed to close down without restriction.

6. Condition for an nth Derivative. We shall now discuss a condition for the mere existence of a derivative.

THEOREM 3. The existence of a unique limit for $A_{n, k}$ as one of the points of $S_{k}$ remains at a, while the rest close down on $a$, is a necessary and sufficient condition for the existence of an nth derivative, $f^{(n)}(a)$.

* Karl Menger, Mathematische Annalen, vol. 103 (1930), p. 480. 
With the restriction stated, by the reasoning previously used for (13) and (16), we see that an alternative statement of the condition for the existence of $f^{(n)}(a)$ can be given in terms of the existence and continuity of $v^{(n-1)}(a)$, where

$$
v(x)=\frac{f(x)-f(0)}{x} .
$$

That the continuity of $v^{(n-1)}(a)$ insures the existence of $f^{(n)}(x)$ follows from (18) and from (20) with $N=n-1$.

To show the converse, we note that $v(0)=f^{\prime}(0)$, and assume that if $f^{(n+1)}(0)$ exists,

$$
v^{(n)}(0)=\frac{f^{(n+1)}(0)}{n+1},
$$

as the basis of an induction. We deduce from (36) that

$$
\begin{aligned}
v^{(n)}(x)=f^{(n)}(x) x^{-1} & -n f^{(n-1)}(x) x^{-2}+\cdots \\
& +(-1)^{n} n ![f(x)-f(0)] x^{-n-1} .
\end{aligned}
$$

Thus

$$
\begin{aligned}
\frac{v^{(n)}(x)-v^{(n)}(0)}{x} & =\frac{f^{(n)}(x) x^{n}-n f^{(n-1)}(x) x^{n-1}}{x^{n+2}}+\cdots \\
+ & \frac{(-1)^{n} n ![f(x)-f(0)]-x^{n+1} f^{(n+1)}(0) /(n+1)}{x^{n+2}} .
\end{aligned}
$$

By L'Hospital's rule, the application of which telescopes the terms, this will approach a limit if

$$
\frac{f^{(n+1)}(x) x^{n}-f^{(n+1)}(0) x^{n}}{(n+2) x^{n+1}}=\frac{f^{(n+1)}(x)-f^{(n+1)}(0)}{(n+2) x}
$$

does. As the latter expression approaches $f^{(n+2)}(0) /(n+2)$, if this derivative exists, we see that in this case

$$
v^{(n+1)}(0)=\frac{f^{(n+2)}(0)}{(n+2)}
$$

which completes our induction.

This proves that if $f^{(n+1)}(0)$ exists, $v^{(n)}(0)$ exists. In this case, in some neighborhood of $0, f^{(n)}(x)$ exists and is continuous. Thus, 
from $(38), v^{(n)}(x)$ exists, and is continuous except perhaps at $x=0$. To settle this point, we write

$$
\begin{aligned}
v^{(n)}(x) & -v^{(n)}(0)=f^{(n)}(x) x^{-1}-f^{(n)}(0) x^{-1}+f^{(n)}(0) x^{-1} \\
& -n f^{(n-1)}(x) x^{-2}+\cdots+(-1)^{n} n ![f(x)-f(0)] x^{-n-1} \\
& -f^{(n+1)}(0) /(n+1) .
\end{aligned}
$$

The limit of the first two terms is $f^{(n+1)}(0)$. The remaining terms may be written with denominator $x^{n+1}$, and their limit found by L'Hospital's rule as for (39). Here we find the limit is that of

which is

$$
\frac{n f^{(n)}(0) x^{n-1}-n f^{(n)}(x) x^{n-1}-f^{(n+1)}(0) x^{n}}{(n+1) x^{n}},
$$

$$
\lim \left[-\frac{n}{n+1} \frac{f^{(n)}(x)-f^{(n)}(0)}{x}-\frac{f^{(n-1)}(0)}{n+1}\right]=-f^{(n+1)}(0)
$$

Thus, from (41),

$$
\lim \left[v^{(n)}(x)-v^{(n)}(0)\right]=0,
$$

and the continuity of $v^{(n)}(x)$ for $x=0$ is established. This completes the proof of Theorem 2.

As a simple example of the theorem, we have the function

$$
f(x)=x^{4} \sin \frac{1}{x}, \quad x \neq 0, \quad f(0)=0,
$$

which has a second derivative, discontinuous for $x=0$. The corresponding $v(x)$ is

$$
v(x)=x^{3} \sin \frac{1}{x}, \quad x \neq 0, \quad v(0)=0,
$$

which has a continuous first derivative.

We formulate in the following corollary the alternative condition used above.

Corollary. A necessary and sufficient condition for the existence of an nth derivative, $f^{(n)}(a)$, is the existence of an $(n-1)$ th derivative, $v^{(n-1)}(x)$, continuous in some neighborhood of $a$, where $v(x)$ is related to $f(x)$ by (36).

Massachusetts Institute of Technology 